\title{
Device Status
}

National Cancer Institute

\section{Source}

National Cancer Institute. Device Status. NCI Thesaurus. Code C160943.

The current status of the device. 